\title{
Application of Pulsed Digital Oscillators to the in Situ Testing of MEMS Vacuum Packaging
}

Surname/firstname: Ricart Jordi, Pons Joan, Domínguez Manuel

Company: Technical University of Catalonia

Address: Jordi Girona 1-3, Barcelona, CP08034, SPAIN

Abstract- The purpose of this work is to show that Pulsed Digital Oscillators can be used to monitor the working pressure of MEMS with vacuum packaging. The oscillation frequency can be directly recovered from the bitstream at the output of the oscillator. Experiments are shown on which a MEMS cantilever is subjected to different pressure conditions, and these changes are tracked directly from the bitstream.

\section{INTRODUCTION}

There is a large number of applications where the use of MEMS structures under low pressure conditions exhibits obvious advantages [1-5]. In the specific case of high-Q MEMS resonators, much effort has been directed towards obtaining devices with very low pressure leakage. However this issue clearly affects the reliability of nowadays MEMS working under vacuum or high vacuum conditions. The main purpose of this work is to show the application of Pulsed Digital Oscillators to the in situ detection of pressure leaks in vacuum packaged MEMS structures.

Pulsed Digital Oscillators are simple nonlinear circuits that can overcome some of the usual nonlinealirities in MEMS actuation and sensing [6-8]. PDOs are sampled circuits and at each sampling time, they only need to know whether the resonator is above or below its rest position (see Figure 1). The actuation force fed into the resonator consists of a train of short pulses of force, the output of the 1-bit quantizer.

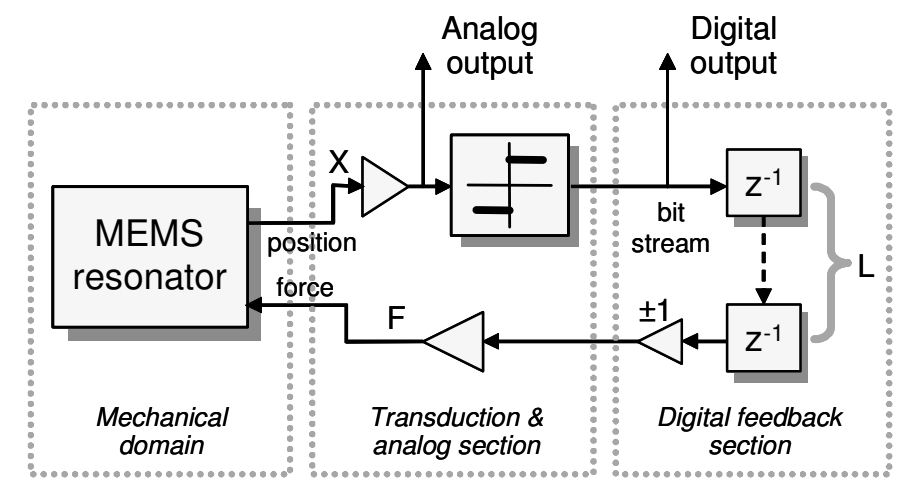

Fig. 1. Schematic diagram of a PDO with L delays in the feedback loop.

Although PDOs are very similar to sigma-delta modulators, their dynamics is very different [8]. It has been proved that, under some conditions, these circuits can produce at their digital output some sequences $b_{n}$ called the Sign of a Sampled Sinusoid, briefly $S^{3}$, sequences

$$
b_{n}=\operatorname{sign}(\sin (2 \pi f n+\theta)), n \geq 0
$$

for some normalized digital oscillation frequency $f$ and phase $\theta$. For resonators with very low damping losses, $f=f_{0} / f_{S}$, being $f_{0}$ is the mechanical resonant frequency of the MEMS and $f_{S}$ the sampling frequency. The bidirectional conversion between $S^{3}$ sequences and 'classical' first-order sigma-delta sequences can be easily done using the circuits shown in Figure 2. In this way, an all-digital inference of the oscillation frequency of the MEMS resonator can easily be obtained through the standard tools of sigma-delta modulation: filtering and decimation.

Moreover, within this framework detection of pressure leaks in vacuum sealed MEMS that generate frequency shifts becomes possible and easy. So the purpose of this work is to show how changes in pressure conditions affect the resonant frequency of a MEMS cantilever, and to show that these changes can be easily monitored in PDO-based systems using digital filtering and decimation. The experimental setup is explained in Section II, whereas the experimental results obtained so far are shown in Section III. 


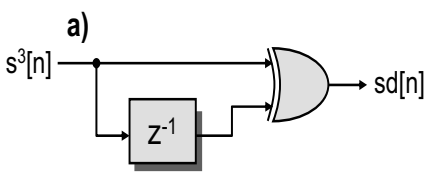

b)

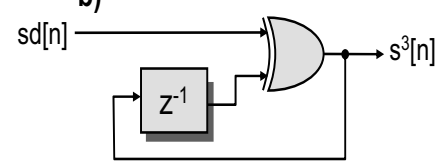

Fig. 2. Conversion circuits between $S^{3}$ and first-order sigma-delta (SD) sequences. a) from $S^{3}$ sequences to $S D$ and $b$ ) from $S D$ to $S^{3}$. If the $S^{3}$ sequence has a frequency $f$, then the $S D$ sequence has a parameter $2 f$.

\section{EXPERIMENTAL SETUP}

\section{1 MEMS devices}

The MEMS resonators used in our experiments are from a set of silicon cantilevers that were originally designed for chemical sensing purposes [6-8], and that have been extensively tested with PDOs. Each cantilever has a squared plate, of $300 \mu \mathrm{m}$ long, $300 \mu \mathrm{m}$ wide and $5 \mu \mathrm{m}$ thick. The plate is held by three arms of $100 \mu \mathrm{m}$ long.

The structure that is used to sense the cantilever movement is located in the middle arm, being the actuation layers in the two external arms. The actuation mechanism is thermoelectric: it generates a force in the cantilever which is proportional to the heat dissipated in two resistors, therefore being proportional to the square of the applied voltage. This non-linear actuation mechanism poses a strong drawback for standard large signal oscillators, because i.e. assuming a sinusoidal waveform excitation the frequency is doubled in the feedback loop. To overcome this problem, the PDO generates short pulses of force of the same amplitude, thus non linearity in actuation becomes not relevant.

On the other hand, MEMS position sensing is achieved through a piezoresistive Wheatstone bridge located in the middle arm of the cantilever. Although a PDO structure only needs to know the sign of the position samples, in the particular case of the experimental measurements for this work, the MEMS position (i.e. analog output in Fig. 1) has been extracted in order to monitor the position behavior and as an additional way to extract the PDO oscillation frequency.

\section{II.2 Experimental Setup}

Figure 3 depicts an schematic of the measurement setup components. Both the MEMS resonator and the small set of components that makes the transduction and the analog functions of the PDO are located within a sealed camera. Pressure ranges between $1 \mathrm{mbar}$ and 1 bar have been used on measurements.

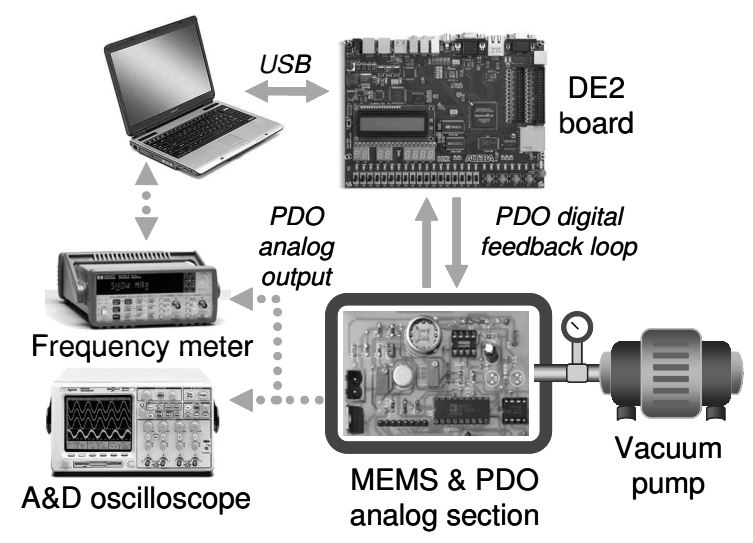

Fig. 3. Experimental setup. Two simultaneous frequency measurements are performed, one extracted from the MEMS position (i.e. analog), and a second one extracted from the PDO bit stream (i.e. digital).

To implement the prototypes of the PDO digital section, an Altera-Terasic development and education DE-2 board, based on a FPGA of the Cyclone II family from Altera, is used. Besides implementing the PDO feedback 
loop, the DE2 board is used to obtain and to store the bit stream samples extracted from the PDO digital output, and, by means of an USB connection, it is also used to send these data to a personal computer.

As commented above, in addition to the PDO digital output our measurement prototype also extracts and processes analog data of the MEMS position extracted from the PDO analog output. Thus, the oscillation frequency of the MEMS device is extracted here by means of a 531A Universal Counter, whereas the waveforms are registered using an Agilent 54621D mixed analog-digital oscilloscope.

\section{II.3 Digital Control section}

It is obvious that the practical implementation of the digital section of a PDO destined to a specific application, such as the one that is the objective of this work, implies to use a very reduced amount of logical resources (see Fig. 1). Thus, a DE2 board is, at first sight, a too large solution to implement such digital section, but it is a comfortable enviroment for prototyping purposes, including facilities for data communication and storing.

Two different methods of capturing and storing the samples extracted from the PDO digital output have been implemented and used:

- Bit Stream Method. Each 16-bit memory word contains 16 consecutive bit samples coming directly from the PDO digital output.

- Event Count Method. Each 16-bit memory word contains the total number of rising and falling events obtained in $2^{16}$ consecutive bit samples coming directly from the PDO digital output.

The second method is a straightforward application of the theory shown in [8] and implies a conversion of the original PDO bit stream into a sigma-delta sequence (see Figure 2). This method provides several advantages because it makes a fast data preprocessing on the acquisition board and it allows a high efficiency on memory usage (which is equivalent to a spectacular increase of the time duration of the measurement captured in memory): the bit stream method and the SRAM size allow storing a maximum of $2^{18} \times 16$ samples, while the event count method allows storing a maximum of $2^{18} \times 2^{16}$ samples.

\section{EXPERIMENTAL RESULTS AND DISCUSSION}

An extensive set of measurements has been carried out. The first measurements have been obtained in oversampling conditions, i.e. with sampling frequency ratio values slightly above the Nyquist limit $f_{0} / f_{S}=0.5$. Let us note that this should not be confused with standard sigma-delta modulation oversampling, where ratios of the order of 7-10 between the bandwidth of the signal to be converted and the sampling frequency are often used. Another set of measurements was done in undersampling conditions. PDOs, working with sampling frequencies below the Nyquist limit imply that the oscillation frequency cannot be perfectly recovered from the bitstream, but that small changes in the oscillation frequency can be easily monitored.

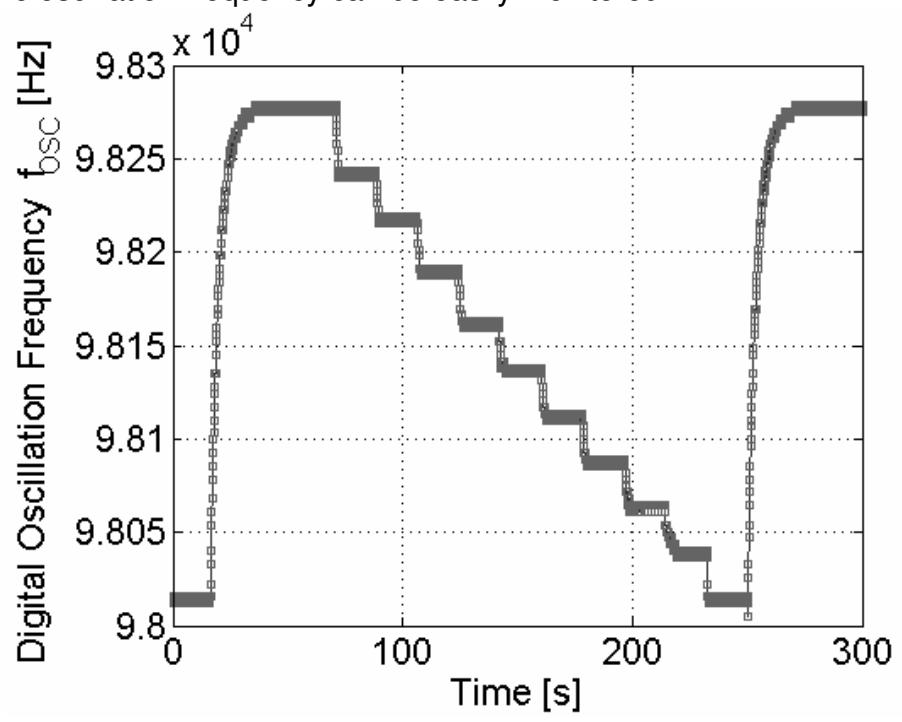

Fig. 4. Digital oscillation frequency after stepped pressure transients, obtained from the PDO digital output using the event count method. Bottom value corresponds to room pressure (1 bar), while top value corresponds to high vacuum (below $1 \mathrm{mbar}$ ). Increasing steps of $100 \mathrm{mbar}$ are applied until room pressure is restored. PDO parameters: $L=2$ delays, $f_{S}=460 \mathrm{kHz}$ (over sampling). 
Different PDO structures, with different feedback filters and different sampling frequencies have been tested. Figure 4 shows the oscillation frequency of the PDO, working at a sampling frequency of $460 \mathrm{KHz}$, and with a feedback filter composed of 2 delays. As it can be observed, the resonant frequency decreases with increasing pressure. Steps of 100 mbar are applied down to a final pressure of $1 \mathrm{mbar}$.

Results shown in Figure 4 have been obtained directly from the bitstream at the output of the PDO, with the event count method. On the other hand, Figure 5 shows the oscillation frequency obtained with a frequency meter from the analog waveform at the output of the Wheadstone bridge of the MEMS cantilever. As it can be observed both results are almost identical, and therefore the capability of obtaining the oscillation frequency directly from the bitstream of the PDO has been demonstrated.

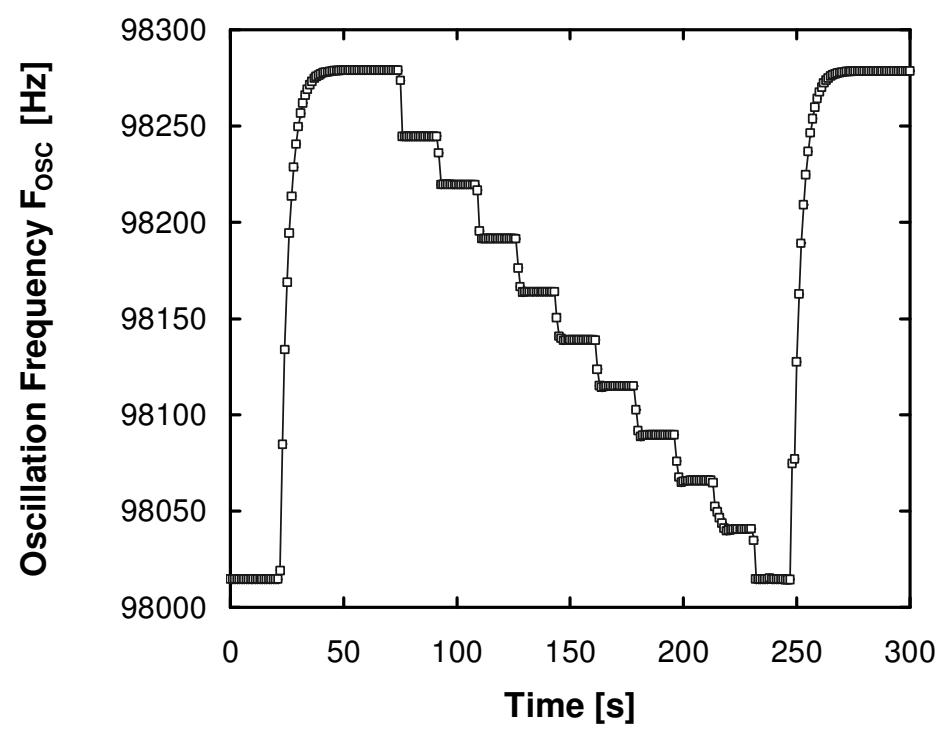

Fig. 5. Oscillation frequencies obtained from the PDO analog output after stepped pressure transients. Experimental conditions are the same as in Fig. 4.

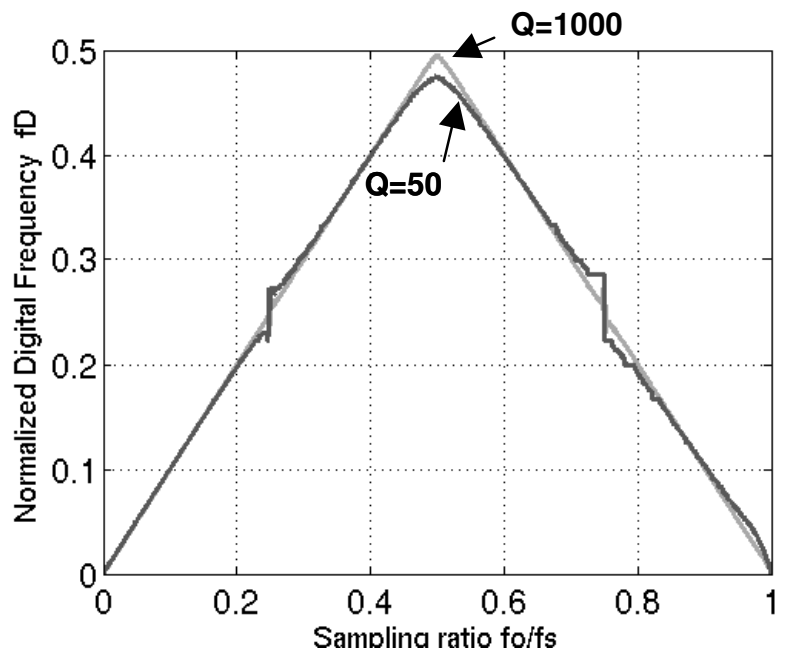

Fig. 6. PDO normalized digital frequency, $f_{D}=f_{O S C} / f_{S}$, versus sampling ratio for two different MEMS quality factors (i.e. two values of damping losses) and $L=2$. For sampling ratios under the Nyquist limit (fo/f $\left.f_{S}>0.5\right)$, aliasing exists and the diaital frequency obtained is different than the actual PDO oscillation frequencv. $L=2$ delavs

PDOs, given a resonator, a feedback filter and a sampling frequency either in average provide energy to the resonator, or extract it until a limit cycle is reached near the origin [9]. For the two-delay feedback filter used in Figures 4 and 5, the oscillation frequency depends on the damping losses of the mechanical resonator, and on the sign of the feedback filter. Figure 6 shows the simulated oscillation frequency for this feedback filter, for a 
MEMS resonator with two different quality factors, $Q$. In this case, in order to avoid the reverse mode [10] it is necessary to change the sign of the feedback filter for sample ratios in the range $0.25-0.75$. For high losses the discontinuity between the two regimes can be clearly observed.

As commented above, a second set of measurements below the Nyquist limit has been done. In this case, it is not possible to fully recover the oscillation frequency directly from the bitstream. However, a rough beforehand knowledge of the spectrum region on which the oscillation frequency is present allons to obtain its value after a simple frequency shift [10].

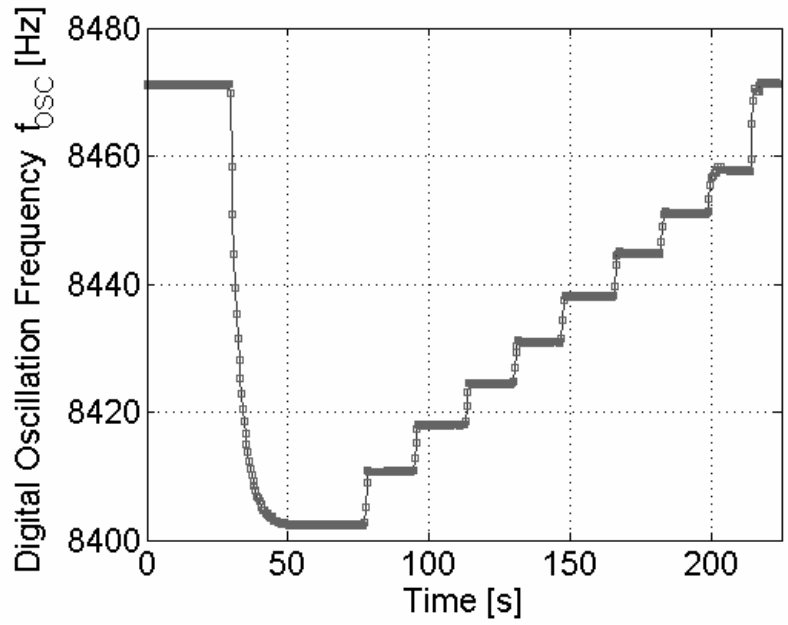

Fig. 7. Digital oscillation frequency after stepped pressure transients, obtained from the PDO digital output using the event count method. Top value corresponds to room pressure (1 bar), while bottom value corresponds to high vacuum (below $1 \mathrm{mbar}$ ). Increasing steps of $100 \mathrm{mbar}$ are applied until room pressure is restored. PDO parameters: $L=1$ delays, $f_{S}=128.7 \mathrm{kHz}$ (under sampling).

Figure 7 shows the digital oscillation frequency (i.e. obtained from the bitstream) for a PDO system working in undersampling conditions. It can be seen that the digital oscillation frequency, in this case, decreases with decreasing vakues of damping. This effect is due to the PDO frequency response shape seen in Figures 6 or 8 . Figure 8 shows a simulation similar to that of Figure 6 but for a PDO with a one delay filter. The sign of the feedback loop must be changed from 0.5 to 1 , and the abrupt transitions present in Figure 6 , at $f=0.25$ or 0.75 , can no longer be seen. However for both cases and in the undersampling region between $f=0.5$ and $f=1$, the digital oscillation frequency decreases with decreasing damping: note the difference between both curves for different damping values.

Although in Figure 7 the extracted digital oscillation frequency is in the range of $8.4 \mathrm{KHz}-8.5 \mathrm{KHz}$, the oscillation frequency directly extracted from the analog waveform at the output of the Wheatstone bridge of the MEMS cantilever shows that in fact the resonator is oscillating near its resonant frequency (see Figure 9). This way, it is possible to infer small changes of the resonant frequency of the resonator, even working far below the Nyquist limit.

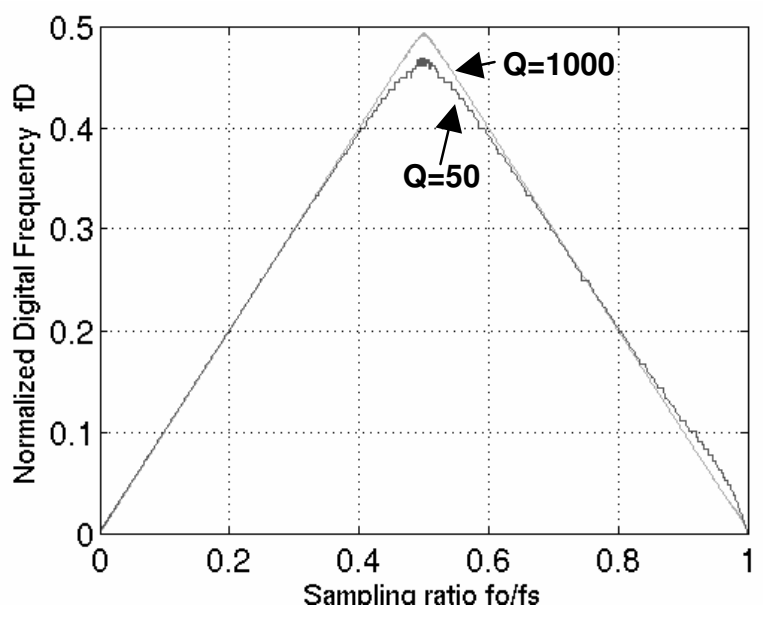

Fig. 8. PDO normalized digital frequency, $f_{D}$ versus sampling ratio for two different MEMS quality factors and $L=1$. 


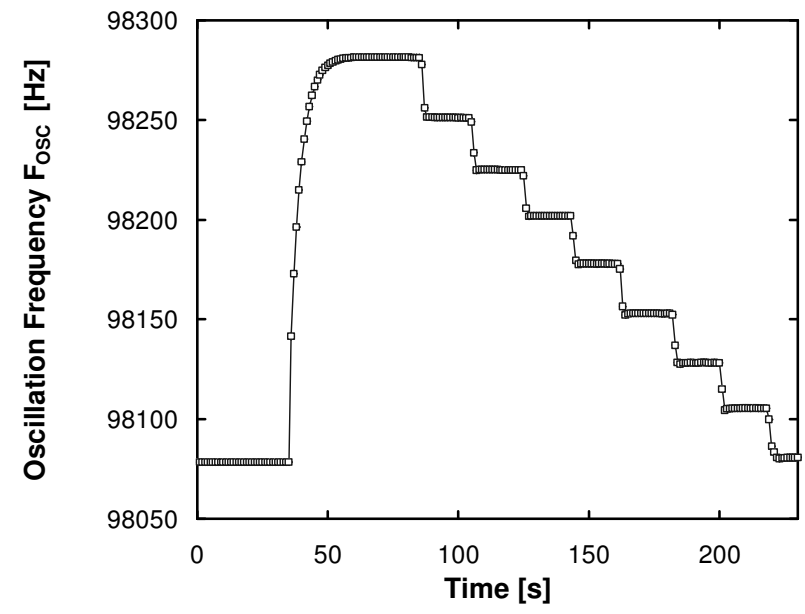

Fig. 9. Oscillation frequencies obtained from the PDO analog output after stepped pressure transients. Experimental conditions are the same as in Fig. 7.

\section{CONCLUSION}

It has been experimentally proved that the oscillation frequency of the MEMS resonator can be recovered from the bitstream at the output of PDOs. By carrying out a simple operation in the output bitstream another is obtained with the typical noise-shaping characteristic of sigma-delta modulation. This allows a simple and all-digital treatment of the resonant frequency of MEMS resonators, with standard tools of sigma-delta modulation, filtering and decimation. This procedure allows to monitor the pressure conditions of a MEMS resonator in vaccum packaging.

\section{REFERENCES}

[1] Doms M., Muller J., "A Micromachined Vapor-Jet Vacuum Pump", Solid-State Sensors, Actuators and Microsystems Conference, 2007. TRANSDUCERS 2007, pp. 2425 - 2428, June 2007

[2] V. Kaajakari, J. Kiiham ki, A. Oja , S. Pietikäinen, V. Kokkala, H. Kuisma,"Stability of wafer level vacuum encapsulated single-crystal silicon resonators", Sensors and Actuators A, pp. 42-47, 2006

[3] Y. Tao, A. P. Malshe, W.D. Brown, D.R. DeReus,S. Cunninghame,"'Laser-Assisted Sealing and Testing for Ceramic Packaging of MEMS Devices", IEEE Trans. Adv. Packaging, vol. 26, num. 3, 2003

[4] W.T. Hsu, "Low Cost Packages for MEMS Oscillators", IEMT '07. 32nd IEEE/CPMT International, pp. 273-277, Oct. 2007

[5] F. Völklein, A. Meier, "Microstructured vacuum gauges and their future perspectives", Vacuum, vol. 82, pp. 420-430, 2008

[6] M. Domínguez, J. Pons, J. Ricart, A. Bermejo, E. Figueras, "A Novel Sigma-Delta Pulsed Digital Oscillator (PDO) for MEMS", IEEE Sensors Journal, Vol. 5, pp. 1379-1388, 2005.

[7] M. Domínguez, J. Pons, J. Ricart, A. Bermejo, E. Figueras, "Analysis of the $\Sigma-\Delta$ Pulsed Digital Oscillator (PDO) for MEMS", IEEE Trans. on Circuits and Systems I, Vol. 52, pp. 2286-2297, 2005

[8] M. Domínguez, J. Pons, J. Ricart, "General Dynamics of Pulsed Digital Oscillators", IEEE Trans. on Circuits and Systems I, Vol. 55, No. 7, pp. 2038-2050, 2008.

[9] M. Domínguez, J. Pons, J. Ricart, E. Figueras, "The MEMS Pulsed Digital Oscillator (PDO) below the Nyquist limit," Sensors and Actuators Phys. A, Vol. 136, pp. 690-696, 2007.

[10] M. Dominguez, J. Pons, J. Ricart, "Application of Pulsed Digital Oscillators in reverse mode to eliminate undesired vibrations in high-Q MEMS resonators", Proc. ISCAS-2007. 